\title{
The Main Factors Responsible for Self-Injury among Adolescents
}

\author{
Jackson de Carvalho, PhD \\ Associate Professor \\ Prairie View A \& M University \\ Prairie View, Texas 77446 \\ United States of America \\ Darron Garner, PhD \\ Department Head for Human Services \\ Alvin Community College \\ Houston, Texas \\ United States of America
}

\begin{abstract}
This study focuses on the main factors responsible for self-injurious behaviors and examines its association to dissociative phenomena. Self-injury has been conceptualized from a functional perspective and defined in this study as the behavior of purposefully inflicting superficial to moderate damage to the body without suicidal intent. Dissociation is a natural defense mechanism that can help protect ourselves from unbearable painful emotional and or psychological experience. Self-injury is subsequently utilized as a coping skill to regulate internal emotions, while decreasing pain and discomfort. Motivations can range from a form of self-punishment, to the release of negative feelings, stress management, the expression of anger, an increased sense of mastery or control, and management of dissociative processes. Thus, the purpose of educating ourselves on self-injurious behaviors is to become aware and confident in our ability to handle such a complex phenomenon.
\end{abstract}

Keywords: Self-injury, adolescents, factors and functions.

\section{Introduction}

The remediation of psychological distress and the elimination of maladaptive strategies of coping is a primary goal of mental health professionals. This study assessed the relationship between self-injury and dissociative experiences perpetuated by the motivational functions of self-injurious behaviors. According to Klonsky and Moyer (2008), those who regularly self-injure do so as a means of regulating their emotions, cognitions and social environments. In fact, it has been identified that many of these individuals experience varying degrees and types of dissociative experiences (Gratz, 2007; Muehlenkamp, \& Gutierrez, 2007).If a relationship does exist between self-injury and dissociation, then new or specialized treatment interventions may be indicated for this clinical population.

Mental health practitioners are increasingly encountering adolescents and adults who report using self-injury such as, cutting, scratching, picking wounds, etc. as a means of coping with stress. Individuals who engage in selfinjury are often triggered by internal distress and external stressors (Gratz, 2006; Polk \&Liss, 2007). The regulation of emotional and cognitive states via self-injury often produces feelings of guilt or shame. Selfinjurious adolescents often report they clung to and used self-injury because it offered temporary relief from depression, anxiety, and anger. Unfortunately, self-injury appears to be a particularly difficult behavior to treat. There are multiple variables and etiological factors which contribute to self-injury and dissociation; these factors have been evaluated and discussed at length in this paper (Walsh, 2006).

This paper focuses on the functions of self-injury and its association to dissociative experiences among adolescents struggling to cope with stressful challenges of a rapidly changing society. Dissociation has been broadly defined as a disruption of consciousness, memory, identity, or perception of environment (APA, 2013). Dissociation is a natural human phenomenon that can be conceptualized on a continuum, ranging from "normal" to "pathological." 
It is believed that the majority of individuals who engage in habitual self-injury may have a natural inclination or predisposition to dissociate more readily than the "average" person (Calati\& Philippe, 2016). If this is true, then individuals who self-injure may have an increased ability to utilize dissociative laden therapeutic techniques, such as hypnosis, meditation, or Eye Movement Desensitization and Reprocessing (EMDR). The assessment of the relationship between self-injury and dissociation could be particularly helpful in several ways. First and foremost, it may help clinicians to more fully understand the extent to which youths who struggle with self-injury experience dissociative phenomena. Additionally, the identification of an individual's level of dissociation may give clinicians outcome predictability regarding how receptive the client may be to a course of treatment. Furthermore, clinicians could provide psycho education to self-injury patients about dissociation, in the hopes that it would reduce fear and alleviate anxiety about experiencing these phenomena. Finally, clinicians could design specialized treatment based upon the function(s) of self-injury considering a patient's reported severity and type of dissociation (Trepal\& Wester, 2007).

\section{Literature Review}

An extensive review of the current psychological and psychiatric literature revealed the absence of a conclusive definition of self-injury; instead multiple terms are used to describe self-injurious behavior. Despite the complication inherent in the use of multiple terms to describe the same phenomenon, a consensus regarding the parameters of self-injurious behavior has emerged. The terminological quagmire for describing self-injury is evidence of the complexity of this psychological phenomenon; where the absence of a regulatory body making proclamations regarding endorsed labels for self-injury has created a free forum for term usage and development. The presence of multiple definitions also bears witness to the mental health field's continued evolution, i.e.

Labels have changed or been modified as research findings are generated. The emergence of self-injury as a primary focus of treatment reflects an awaking within the mental health field of the growing need to help individuals who struggle with these maladaptive behaviors. Consequently, self-injury will be defined in this study as the behavior of purposefully inflicting superficial to moderate damage to the body tissue; it denotes the absence of suicidal intent and is considered socially unacceptable (Landsverk, Burns, Stambaugh, \& Rolls, 2007; Klonsky, 2007).

Not incorporated into the definition of SIBS, but essential to understanding self-injury, are the array of motivational factors, or functions, these behaviors can simultaneously serve. Although not mutually exclusive, current literature suggests that acts of self-injury can serve as both an internal regulatory factor, while also possessing external communicative value. Motivations can range from a form of self-punishment, to the release of negative feelings, stress management, the expression of anger, and an increased sense of mastery or control, and management of dissociative processes (Conradi \& Wilson, 2010; Gratz \& Chapman, 2007; Nock \& Prinstein, 2005).

After reviewing firsthand accounts of individuals who self-injure, it is suggested that many engage in acts of selfinjury to reduce psychological distress. By harming themselves, individuals attempt to reduce their emotional and psychological arousal to a bearable level. Many self-injurious adolescents report mounting tensions that they cannot control, while others wish to escape from feelings of emptiness, depression, or the inability to experience normal sensations. The challenge of defining self-injury unfortunately has produced unintentional negative ramifications for researchers as they have sought to determine the scope and frequency of this problem behavior. Researchers must be cognizant of how each study defines self-injury to avoid poor scholarship (Calati \& Philippe, 2016; Conradi\& Wilson, 2010).

To date, epidemiological studies on self-injury in both clinical and non-clinical samples have revealed varying degrees of prevalence. One of the most frequently cited studies on the prevalence of self-injury is Laye-Gindhu and Schonert-Reichl (2005), which collected data from 424 adolescents (236 girls, 188 boys), mean age 15.34 years, who attended a public high school. The study reported that 15\% of the sample, 64 adolescents (48 girls, 16 boys) engaged in self-harming behavior. The authors used the term self-harm because they wanted to report on a broader range of behaviors which included suicide attempts, tattooing, general recklessness, and specific acts of self-injury, e.g. cutting, burning, and scratching.

The prevalence of self-injury in male and female undergraduate college students has recently been assessed as well (Gratz \& Chapman, 2009; Gratz 2006). In a sample of 97 college men, Gratz and Chapman (2007) found that $44 \%(n=43)$ reported a history of engaging in deliberate self-harm. 
The most frequent self-harming behaviors included cutting (30\%), burning with a lighter/match (30\%), and burning with a cigarette (28\%). In a similar research design, Gratz (2006) found that out of a sample of 249 female college students, 37\% $(\mathrm{n}=91)$ reported a history of self-harm. Somewhat similar to the men's study, Gratz (2006) found that the most common method of self-harm women used included skin-cutting (46\%), severe scratching (34\%), and carving words into one's skin (32\%). These recent studies seem to indicate that the prevalence of self-injury histories among college students is surprisingly high for both males and females (Conradi\& Wilson, 2010).

Gender differences in self-injury may stem from an actual difference, i.e. women "prefer" acts of self-injury while men "prefer" other forms of self-harm, e.g. substance abuse. On the other hand, the apparent gender difference could be related to reporting bias and cultural influences, e.g. it is more "acceptable" for women to engage in selfinjury and it is more "acceptable" for to engage in substance abuse. Gender differences appear most prominent and stable within adolescence samples, in which girls seem to be a greater risk for self-injury (Katz, 2008). Despite the apparent gender difference found in some studies, clinicians would be wise to shed long standing stereotypes that have dominated the mental health field, specifically the belief that women are significantly more likely to utilize self-injurious behaviors than men (Conradi\& Wilson, 2010).

\section{Etiology of Self-injurious behavior}

The current research study literature shows several reasons for the presence of self-injurious behaviors. While a pure understanding of the etiology of self-injury remains vague, research and clinical observations have identified many of the foundational pillars of this psychological phenomenon. Each pillar appears to uphold and perpetuate this problem behavior (Klonsky \&Muehlenkamp, 2007). Higher levels of predicting factors are associated with higher levels of problem behaviors, which makes challenging for clinicians to intervene successfully. The identification of levels of factor allow to measure the impact of "determinates" to the individual's psychological make-up into one's personality while at the same time providing insight for the external behavior evidenced on the body.

The most frequently referenced factor or life event that is strongly associated with self-injury is child abuse or maltreatment, which typically includes both physical and sexual abuse (Washburn, Richardt, Styer, Gebhardt, Juzwin, Yourek, \&Aldridge,2012; Gratz \& Chapman, 2007; Gratz, 2006). Abuse history serves as a developmental block which inhibits the formation of basic trust. Consequently, the child's developmental trajectory is altered, which in turn may negatively impact all or several domains of later development. Research conducted by van der Kolk, Perry, \& Herman, 1991; identified that individuals who had a diagnosis of Posttraumatic Stress Disorder (PTSD) due to a history of childhood abuse, often had three commonly occurring negative psychological symptoms: dissociation, somatization and affect dysregulation. Similarly, Washburn, Richardt, Styer, Gebhardt, Juzwin, Yourek, \& Aldridge, (2012) found that adolescents diagnosed with PTSD and who had experienced sexual abuse were uniquely disposed to use Self-injury as a means of managing reexperiencing symptoms and numbing symptoms.

Furthermore, Landsverk, et al. (2009) identified that patients labeled as "hysterics" frequently displayed violence against themselves or others; these patients complained of somatic conditions and experienced dissociative problems. The authors noted that this cluster of psychological symptoms is strongly correlated with childhood history of abuse, particularly when the trauma is early and prolonged. A similar psychological factor that may predict self-injuries behavior is recent trauma. According to Mojtabai and Olfson (2008), self-cutting may appear as a behavioral symptom of PTSD. Specifically, Webb (2006) reported that in their clinical experience, selfcutting frequently occurred in rape victims. The authors provided three case studies, two of which were victims who did not have any history of abuse nor a personality disorder prior to being raped, while the third patient did have a previous history of abuse and a personality disorder. In all three cases, the onset of self-cutting occurred after being raped.

Another predicting factor which appears to have a strong correlation with self-injurious behaviors is the presence of emotional in expressivity. According to Gratz (2006) an individual's inability or reluctance to express negative affect may cause the person to stuff or bury his or her feelings, which, later is expressed through acts of deliberate self-harm. In an earlier paper, Gratz (2003) asserted that emotional neglect was the strongest predictor of deliberate self-harm. While more recently, Gratz and Chapman (2007) found that college males reported using deliberate self-harming behaviors due to its emotional regulatory effects, which appeared to operate or function independently from histories of childhood abuse. 
The findings by Gratz (2006, 2003) and Gratz and Chapman (2007) are in alignment with Linehan's (1993) conceptualizations pertaining to how emotionally stressed family environments are significantly correlated with borderline personality disorder and self-injurious behavior. In their study of individuals who presented to a Scottish Hospital's emergency department for self-injury, O'Connor et al. (2006) discovered that depression and lack of self-efficacy were the strongest predictors of deliberate self-harm. In particular, the researchers noted that the beliefs and behaviors of friends and peers about deliberate self-harm, had a considerable impact upon a patient's intention to engage in self-harming behaviors. The study findings by O'Connor et al. (2006) seems to support the notion that social connectivity and the reciprocal emotional support that it provides may have a mediating effect upon whether an individual will utilize self-injury. Lack of self-efficacy and lack of social support, seem to co-occur, or go together, and each may play a role in the incident and prevention of self-injury. Self-injury is frequently found in individuals diagnosed with a personality disorder, especially Borderline Personality Disorder (BPD). In its current format, the Diagnostic and Statistical Manual of Mental Disorders, fourth edition, text revision (DSM-IV-TR) fifth criterion for BPD is "recurrent suicidal behavior, gestures, or threats, or self-mutilating behavior" (APA, 2013, p. 292). This reference of self-mutilating behavior is the only one in the DSM-IV-TR. Thus, there has been a historical bias to diagnosis individuals who self-injure as having BPD. The strong association between self-injury and BPD persists to this day. Research conducted by Polk and Liss (2007) found that those who were diagnosed with BPD almost exclusively had distinct childhood trauma histories, when compared to individuals who had borderline traits, other personality disorders, or bipolar II disorder. The distinct markers identified by Polk and Liss (2007) for individuals diagnosed with BPD were early childhood trauma (0-6 years), experiencing multiple types of trauma, and trauma which lasted for longer periods of time.

In summary, the epidemiological studies and clinical observations appear to have isolated several of the pillars which support self-injurious behaviors, these include: (a) childhood abuse history, (b) recent trauma, (c) family instability and lack of emotional support, (d) emotional in expressivity, (e) lack of social support and lack of selfefficacy, (f) the presence of a personality disorder, usually BPD, and (g) a stress disorder, usually PTSD which includes dissociation, somatization and affect dysregulation.

\section{Purposes ofSelf-Injury}

Once the etiology of a maladaptive behavior is considered, psychologists typically seek to determine the reasons why a behavior is used. In other words, understanding the historical events which may have contributed to or shaped an individual's current functioning is informative, but it fails to account for the patient's reasoning for why he or she continually utilizes a specific behavior or set of related behaviors. The focus then turns to an evaluation of the functions of behavior, i.e. the reasons why it is used and the effect(s) it produces. Through the dual assessment of etiology and the functions of a behavior, psychologists are in a better position to tailor their interventions to match the unique needs of a patient. In this section, the most commonly referenced theories regarding why patients engage in self-injury is presented.

According to Calati and Philippe (2016), historically, self-injury was considered a symptom of hysteria. However, since the publication of the DSM-III in 1980, the term hysteria was dropped and the diagnostic categories of stress disorders, dissociative disorders, and somatic disorders were established, which served to separate the various symptoms of hysteria into distinct psychological disorders (Mojtabai \& Olfson, 2008). The publication of the DSM-IV in 1994 provided subtle recognition that these distinct disorders may actually be interconnected or related, but it avoided returning to use of the pejorative label, hysteria. In its traditional formulation, hysteria was understood by Pierre Janet to be a reaction to childhood sexual abuse or extreme forms of trauma. A common symptom of "hysterics" as described by Janet and his peers, was the presence of violent reactions against the self or others, called "somnambulistic crises" (Calati \& Philippe, 2016). These early psychological formulations of traumatized patients recognized that self-injury was a common behavioral symptom.

Despite the linking of patient histories with behaviors of self-injury, a full understanding of the function of this behavior and related abnormal behaviors remained elusive. In the past few decades there has been a renewed interest to identify the functions of self-injury, particularly due to the increased reporting and presentation of this problematic behavior to health providers. Affect regulation appears to be the most frequently endorsed reason for self-injury by various patient populations. Nock \& Mendes (2004) identified that the adolescents in their study used self-injury to primarily alleviate depression and tension. Thus, self-injury was viewed as a vehicle for managing negative feelings and emotions. 
Nock \& Mendes (2005) did note however, that most adolescents endorsed multiple reasons for engaging in selfinjury, which reveals how this maladaptive behavior commonly serves multiple patient needs. In a similar study, Katz (2008) found that $60 \%$ of the adolescent patients in their study reported experiencing emotional relief after cutting themselves. This again points to the primacy of using self-injury as a device for mood management. In her detailed interviews with female psychiatric inpatients, aged 18-54, Grantz (2006) observed that females provided several reasons for self-cutting, some of which included: tension release, affect modulation, the discharge of anger, the induction of a pleasurable state, and as a form of communication. While affect regulation is the most commonly referenced function of self-injury, both researchers and patients have identified several additional reasons for the behavior.

The notion that self-injury may be utilized for multiple reasons aligns well with Suyemoto and Macdonald's (2005) analysis of the functions of self-mutilation, which she said tends to serve multiple functions simultaneously. Suyemoto and Macdonald's (2005) formulated six functional models for self-mutilation, which included the environmental model, the anti-suicide model, the sexual model, the affect regulation model, the dissociation model, and the boundaries model. According to Suyemoto and Macdonald's (2005) each model has its corresponding reason. For instance, in the dissociation model, self-mutilation is used as a means of coping with or ending dissociative experiences. In the sexual model, self-mutilation is seen as an attempt to manage sexual conflicts.

Self-injury can be particularly challenging to treat given that a patient may have multiple motivations for why he or she self-injures. For example, a young adult who experienced severe physical abuse during childhood may resort to self-injury due to an ability to alleviate negative emotions related to painful memories, the same ability may serve as a shield that wards off the unpleasant experience of depersonalization or other dissociative states, and the act of cutting likely produces a physiological high due to the release of endorphins (Grantz, 2007). It is noteworthy that Suyemotoa nd Macdonald's (2005) models are similar to Klonsky's (2007) analysis of deliberate self-injury. According to Klonsky's (2007) review of the psychological and psychiatric literature, there is empirical support for the following functions: affect regulation, anti-dissociation, anti-suicide, interpersonal boundaries, interpersonal-influence, self-punishment, and sensation-seeking. Klonsky provides a descriptive statement for each respective function listed above: "To alleviate acute negative affect or aversive affective arousal," "To end the experience of depersonalization or dissociation," "To replace, compromise with, or avoid the impulse to commit suicide;" "To assert one's autonomy or a distinction between self and others," "To seek help from or manipulate others," "To derogate or express anger towards oneself," and "To generate exhilaration or excitement" (Klonsky, 2007; p. 229). Klonsky notes that overlap or co-occurrence of functions may be common for many individuals who self-injure.

Using the four-factor model for describing the functions of self-mutilative behavior, Nock and Prinstein (2004) identified the following categories: automatic-negative reinforcement, automatic-positive reinforcement, socialnegative reinforcement, and social-positive reinforcement. Nock and Prinstein's (2004) model has two dichotomous dimensions: those behaviors that are automatic vs. social and those that are positive vs. negative. The automatic vs. social dimension refers to management of one's internal world compared to management of one's social field. The positive vs. negative dimension refers to the affect the behavior produces. In the negative, something is brought to an end, e.g. to stop bad feelings, or to avoid having to do something unpleasant, whereas in the positive, something is produced, e.g. to feeling something or to gain attention from others.

Nock and Prinstein's (2004) model of self-mutilation lacks the variety of labels, compared to Klonsky (2007) and Suyemoto and Macdonald's (2005) but it appears superior in its ability to more precisely define how the behavior is reinforcing to the individual and whether or not the focus is on internal or external stressors. In their follow-up study with adolescent psychiatric inpatients, Nock and Prinstein (2005) reported that self-mutilation (defined similarly to the author's preferred term of self-injury), was generally performed impulsively: adolescents tended to report little to no physical pain from the act and they rarely used drugs or alcohol. Nock and Prinstein (2005) highlighted that their four-factor model consistently found adolescents who endorsed both automatic and social reinforcement reasons for using self-injury. Similar models for understanding the reasons why patients engage in self-injury include Ross and Heath's (2002) notions that the behavior is primarily driven by anxiety and hostility. According to the anxiety reduction model, self-mutilation is used to release mounting anxiety and feelings of distress. The hostility model, on the other hand, posits that unexpressed feelings of anger produce tension within the individual which eventually are expressed toward a safe target, the self, through the act of self-injury. 
Ross and Heath (2002) commented that tension reduction is the primary goal of both models and the models are typically viewed as being connected or related. Haines and Williams (2003) similarly asserted that individuals who self-mutilate may have poor or limited coping resources and the behavior reflects deficits in problem solving ability.

In a study of 38 psychiatric inpatients, all of whom had a diagnosis of BPD and who had a history of at least five episodes of self-injury, Kemperman, Russ, and Shearin (1997) found that all the patients reported being motivated to use self-injury to ameliorate unpleasant feelings or affect. Patients also reported to Kemperman et al. (1997) that self-injury provided the most relief for anger. While most hostility and anger theories related to self-injury posit that it is a violent or aggressive act directed against the self, Kemperman et al. (1997) alternatively hypothesized that self-injury could actually provide temporary relief of anger, instead of being viewed solely as an act of self-destruction. This perspective appears to emphasize the function of self-injury as opposed to a psychodynamic interpretation of the behavior. It appears fairly certain that most patients who engage in selfinjury are motivated by more than one function.

The primary motivation for self-injury consistently appears to be the regulation of affect. This finding is hardly surprising given the etiological roots or pillars which are common among individuals who struggle with this maladaptive behavior. Given that there are usually multiple motivations for engaging in self-injury and multiple results or consequences, i.e. temporary changes in emotional, social, and physiological states; it is not surprising that self-injury has been labeled as an addictive behavior by some health providers. Nock and Mendes (2008) identified that patients who struggle with self-injury had greater physiological arousal, i.e. skin conductance, on a distress tolerance test. Thus, the authors theorized that self-injury helps to mediate episodic physiological arousal. The act of damaging one's tissue also has been noted to cause the activation of endogenous opiates (Calati and Philippe (2016), which produce a physiological state change in the body. While the addiction model or explanation for self-injury is popular, other researchers believe it is more accurate to conceptualized self-injury as a disorder of impulse control (Gratz \& Chapman, 2009). Regardless of theoretical vantage point, awareness of both the origins and the functions of self-injury are essential for the design and application of thoughtful therapeutic interventions. Of course, this assumes that clinicians have correctly assessed their patients, prior to initiating a regiment of treatment.

\section{Purposeful Categories of Self Injury}

It is noteworthy, that the literature does not single out a specific category of motivations of self-injurious behaviors, instead it suggests that juveniles engage in acts of self-injury driven by several categories of motivations. In fact, Calati and Philippe (2016) explain that motivations can range from a form of selfpunishment, to the release of negative feelings, stress management, the expression of anger, an increased sense of mastery or control, and management of dissociative processes. Moreover, Gratz and Chapman (2009) explain that many individuals engage in acts of self-injury in an attempt to regulate internal emotions and to reduce psychological distress while externally communicating an outcry for help. By harming themselves, individuals may attempt to reduce their emotional and psychological arousal to a bearable level. Many report mounting tensions that they cannot control, while others wish to escape from feelings of emptiness, depression, or the inability to experience normal sensations.

Nevertheless, Nock and Prinstein (2004) conducted a research study employing a sample comprised of 108 adolescents, ages 12-17 within a clinical, inpatient setting and found that individuals who participate in selfinjurious behaviors are motivated by four primary functional categories:

1) Trauma enactment- Although it may seem strange, some trauma survivors desperately try to avoid reminders of the trauma, while simultaneously re-exposing themselves to further victimization in the form of abusive relationships, dangerous risk-taking behaviors, and acts of self-injury. For many trauma survivors, self-injury can be seen as attempts to master a previously unmanageable situation. It is as if the survivor is saying 'This time I will be able to control what happens' or 'This time I'll be in charge of the pain and decide when it's too much. ' Thus, self-injury can be seen as a way of not only gaining control over one's own emotions, but also as an attempt to gain control over one's immediate environment.

2) Expression of Needs and Feelings - Some individuals who engage in self-injurious behaviors do so as a way of expressing themselves. Many may self-injure because they are struggling with powerful emotions they cannot explain, have secret they cannot reveal, or because they think they are flawed and need to be punished. 
In other words, it is a way for some individuals to express painful or intolerable emotions in an external way. '

${ }^{18}$ Additionally, many survivors of abuse and trauma never learned how to express their feelings or have learned as a survival technique that it is not safe. As a result, later on in life, many may continue to feel that they are unworthy of disclosing their true feelings or simply do not know how. Self-injury serves as a vehicle for expressing an array of emotions that have no other outlet. By targeting these feelings through acts of selfinflicted violence, an individual may attempt to punish themselves for past abuse, while simultaneously allowing the internal emotional pain to become externalized. At other times, these acts may be seen as a cry for help or plea for attention.

3) Attempts to Regain Self-Organization and Homeostasis - The goals of infancy are to have primary needs consistently met, to be soothed, and to develop attachments to a primary caregiver. When these early childhood experiences are marked by inadequate care, however, or when trauma is experienced without any intervention, children are often left to fend for themselves and have difficulty learning to calm themselves. Thus, for many, self-injury becomes a venue for which individuals establish themselves in the world around them and achieve a state of homeostasis, which was never properly learned in infancy. Self-injury is primarily seen as a coping mechanism and/or emotional regulating behavior. Although it may sound strange, some individuals who engage in self- injury report that they do it to get relief from overwhelming negative emotions. They report that it helps them tone down their emotions when they are overwhelmed or to feel something when they are feeling empty or numb inside. Individuals who engage in self- injurious behaviors may not having the proper coping skills to deal with pain, similar to an individual who drowns their sorrows in a bottle of vodka or who punches a wall when they are angry. They cannot deal with their emotions and engage in unhealthy coping skills.

4) Management of Dissociative Process- As previously stated, dissociation is not an easily understood component of self-injury, as there has been debate about whether individuals dissociate during self-injury or whether they self-injure to relieve dissociation. Some individuals report engaging in self-injury to stop feeling numb and feel more alive, while other report dissociating during the act. Regardless of the exact function that self-injury serves for an individual, the subsequent psychological relief is usually immediate and highly effective. Accordingly, many individuals allow self-injury to be their primary coping mechanism, making self-injurious behavior resistant to change and difficult to treat.

In reviewing functional categories of self-injurious behaviors, Plante (2007) discussed its motivations as "doing all the wrong things for the right reasons" (p. 47). The author further noted, "Although cutting and other selfinjurious behaviors is clearly a negative and destructive means of achieving these functional goals, the positive nature of the goals themselves must not be overlooked" (p. 47). Overall, individuals who participate in selfinjurious behaviors identify with a number of functional motivations as to why they partake in this type of action.

\section{Implication for Practice}

The primary goal of mental health professionals should be the reduction of psychological distress and the elimination of maladaptive strategies of coping. This study focuses on examining the impact of childhood abuse on dissociation, leading to self-injurious behavior among adults. According to Klonsky (2009) those who regularly self-injure do so as a means of regulating their emotions, cognitions, and social environments, and it has been identified that many of these individuals experience varying degrees and types of dissociative experiences. Thus, specialized treatment interventions should be indicated for this clinical population. Mental health practitioners are increasingly encountering adolescents and adults who report using self-injury as a means of coping with stress.

The findings of the present study can be used to inform both NSSI treatment and future studies. It may be valuable for therapists to know that low coping self-efficacy may accompany the lack of employed coping skills they may observe in clients who engage in NSSI. Rather than simply encouraging clients to try new coping skills, clinicians may be well advised to work with their clients to explore feelings of competence surrounding the new skills. Directions for further studies might include the exploration of reasons behind the associations between dissociation and NSSI and between NSSI and gender. In addition, ways to increase coping self-efficacy for those who self-injure might also be explored. Furthermore, it would be relevant to explore how increasing coping selfefficacy might decrease dissociation. 


\section{References}

American Psychiatric Association (2013). DSM-5 Parent/Guardian-Rated Level 1 Cross-Cutting Symptom Measure. Washington, D.C.: American Psychiatric Association.

Calati, R., and Philippe, C. (2016). Is Psychotherapy Effective for Reducing Suicide Attempt and Non-Suicidal Self-Injury Rates? Meta-Analysis and Meta-Regression of Literature Data. Journal of Psychiatric Research, 79: 8-20.

Conradi, L., \& Wilson, C. (2010). Managing traumatized children: A trauma systems perspective. Current Opinion in Pediatrics, 22, 621-625.

Briere, J., \& Gil, E. (1998). Self-mutilation in clinical and general population samples: Prevalence, correlates, and functions. American Journal of Orthopsychiatry, 68, 609-620.

Gratz, K. L. (2006). Risk factors for deliberate self-harm among female college students: The role and interaction of childhood maltreatment, emotional inexpressivity, and affect intensity/reactivity. American Journal of Orthopsychiatry, 76, 238-250.

Gratz, K. L. (2007). Targeting emotion dysregulation in the treatment of self-injury. Journal of Clinical Psychology, 63, 1091-1103.

Gratz, K.L. and Chapman, A.L. (2009) Freedom from Self-Harm: Overcoming Self-Injury with Skills from DBT and Other Treatments. Oakland, CA: New Harbinger Publications.

Gratz, K. L. (2003). Risk factors for and functions of deliberate self-harm: An empirical and conceptual review. Clinical Psychology: Science and Practice, 10, 192-205

Haines, J., \& Williams, C. L. (2003). Coping and problem solving of self-mutilators. Journal of Clinical Psychology, 59, 1097-1106.

Katz, C. (2008). Early trauma, defense and effects on later development: A discussion of the papers of Anzieu, Mathelin, and Marans, December 2007, at the conference where the wild things are now: Exciting and scary things for children and therapists. Journal of Infant, Child, and Adolescent Psychotherapy, 7, 185-191.

Kemperman, I., Russ, M. J., \&Shearin, E. (1997). Self-injurious behavior and mood regulation in borderline patients. Journal of Personality Disorders, 11,146-157.

Klonsky, E. D., \& Moyer, A. (2008). Childhood sexual abuse and non-suicidal self-injury: Meta-analysis. The British Journal of Psychiatry, 192, 166-170.

Klonsky, E. D., \&Muehlenkamp, J. J. (2007). Self-injury: A research review for the practitioner. Journal of Clinical Psychology, 63, 1045-1056.

Klonsky, E. D., Oltmanns, T. F, \&Turkheimer, E. (2003). Deliberate self-harm in a nonclinical population: Prevalence and psychological correlates. American Journal of Psychiatry, 160(8), 1501-1508

Landsverk, J. A., Burns, B. J., Stambaugh, L. F. , \&Reutz, J. A. (2009) Psychosocial interventions for children and adolescents in foster care: Review of research literature. Child Welfare, 88(1), 49-69.

Laye-Gindhu, A. \&Schonert-Reichl, K. A. (2005). Nonsuicidal self-harm among community adolescents: Understanding the "whats" and "whys" of self-harm. Journal of Youth and Adolescence, 34, 447-457.

Linehan, M. M. (1993). Cognitive-behavioral treatment for borderline personality disorder. New York, NY: Guilford Press

Mojtabai, R., \&Olfson, M. (2008). Parental detection of youth's self-harm behavior. Suicide \& Life Threatening Behavior, 38(1), 60-73.

Muehlenkamp, J. J. \& Gutierrez, P. M. (2007). Risk for suicide attempts among adolescents who engage in nonsuicidal self-injury. Archives of Suicide Research, 11(1), 69-82.

Nock, M. K., \&Prinstein, M. J. (2004). A functional approach to the assessment of self-mutilative behavior. Journal of Consulting and Clinical Psychology, 72, 885-890

Nock, M.K. \&Prinstein, M.J. (2005). Contextual features and behavioral functions of self-mutilation among adolescents. Journal of Abnormal Psychology, 114(1) 140- 146.

O'Conner, R. C, Armitage, C. J., \& Gray, L. (2006). The role of clinical and social cognitive variables in parasuicide. British Journal of Clinical Psychology, 45, 465-481.

Plante, L. G. (2007). Bleeding to ease the pain. Westport, CT: Praeger.

Polk, E. \&Liss, M. (2007). Psychological characteristics of self-injurious behavior. Personality and Individual Differences, 43, 567- 577. 
Ross, S., \& Heath, N. (2002). A study of the frequency of self-mutilation in a community sample of adolescents. Journal of Youth and Adolescence, 31, 67-77.

Suyemoto, K.L., \& Macdonald, M.L. (1995). Self-cutting in female adolescents. Psychotherapy: Theory, Research and Training, 32(1) 162- 171.

Trepal, H. C. and Wester, K. L. (2007). Self-injurious behaviors, diagnoses, and treatment methods: What mental health professionals are reporting. Journal of Mental Health Counseling, 29(4), 363-375.

van der Kolk, B. A. ,Perry, C. , \& Herman, J. L. (1991). Childhood origins of self-destructive behavior. American Journal of Psychiatry, 148, 1665 - 1671

Walsh, B. W. (2006). Treating self-injury: A practical guide. New York: The Guilford Press.

Washburn, J.J., Richardt, S.L., Styer, D.M, Gebhardt, M., Juzwin, K.R., Yourek, A., and Aldridge, D. (2012). Psychotherapeutic approaches to non-suicidal self-injury in adolescents. Child Adolescent Psychiatry Mental Health, 6(14).

Webb, N. B. (2006). Working with traumatized youth in child welfare. New York: The Guilford Press. 